\title{
Dataset Cartography: Mapping and Diagnosing Datasets with Training Dynamics
}

\author{
Swabha Swayamdipta ${ }^{\dagger} \quad$ Roy Schwartz $^{\ddagger *}$ Nicholas Lourie $^{\dagger}$ \\ Yizhong Wang $^{\diamond}$ Hannaneh Hajishirzi ${ }^{\dagger \diamond}$ Noah A.Smith ${ }^{\dagger \diamond}$ Yejin Choi $^{\dagger \diamond}$
}

${ }^{\dagger}$ Allen Institute for Artificial Intelligence, Seattle, WA, USA

†The Hebrew University of Jerusalem, Israel

$\diamond$ Paul G. Allen School of Computer Science \& Engineering, University of Washington, Seattle, WA, USA

\{swabhas, nicholasl\}eallenai.org roysecs.huji.ac.il

\{yizhongw, hannaneh, noah, yejin\}ecs.washington.edu

\begin{abstract}
Large datasets have become commonplace in NLP research. However, the increased emphasis on data quantity has made it challenging to assess the quality of data. We introduce Data Maps-a model-based tool to characterize and diagnose datasets. We leverage a largely ignored source of information: the behavior of the model on individual instances during training (training dynamics) for building data maps. This yields two intuitive measures for each example- the model's confidence in the true class, and the variability of this confidence across epochs-obtained in a single run of training. Experiments across four datasets show that these model-dependent measures reveal three distinct regions in the data map, each with pronounced characteristics. First, our data maps show the presence of ambiguous regions with respect to the model, which contribute the most towards out-of-distribution generalization. Second, the most populous regions in the data are easy to learn for the model, and play an important role in model optimization. Finally, data maps uncover a region with instances that the model finds hard to learn; these often correspond to labeling errors. Our results indicate that a shift in focus from quantity to quality of data could lead to robust models and improved out-ofdistribution generalization.
\end{abstract}

\section{Introduction}

The creation of large labeled datasets has fueled the advance of AI (Russakovsky et al., 2015; Antol et al., 2015) and NLP in particular (Bowman et al., 2015; Rajpurkar et al., 2016). The common belief is that the more abundant the labeled data, the higher the likelihood of learning diverse phenomena, which in turn leads to models that generalize well. In practice, however, out-of-distribution

\footnotetext{
${ }^{*}$ Work done at the Allen Institute for AI.
}

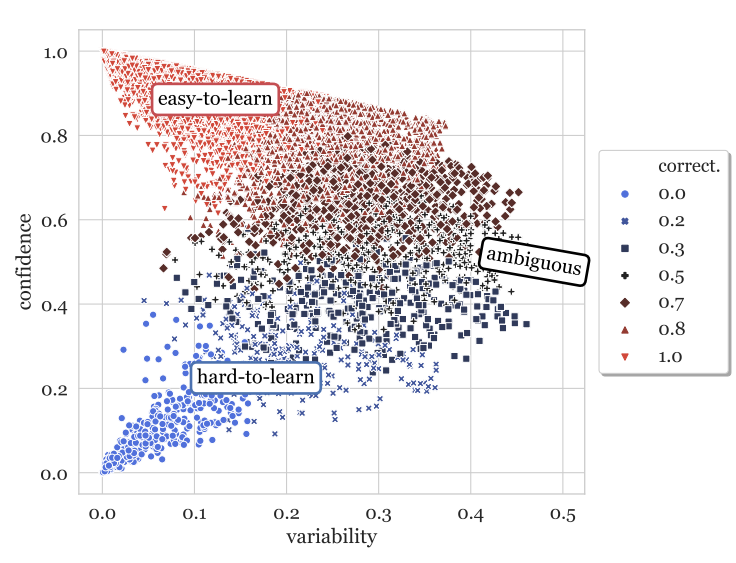

Figure 1: Data map for SNLI train set, based on a RoBERTA-large classifier. The $x$-axis shows variability and $y$-axis, the confidence; the colors/shapes indicate correctness. The top-left corner of the data map (low variability, high confidence) corresponds to easy-to-learn examples, the bottomleft corner (low variability, low confidence) corresponds to hard-to-learn examples, and examples on the right (with high variability) are ambiguous; all definitions are with respect to the RoBERTA-large model. The modal group in the data is formed by the easy-to-learn regions. For clarity we only plot $25 \mathrm{~K}$ random samples from the SNLI train set. Fig. $8 \mathrm{~b}$ in App. $\S \mathrm{C}$ shows the same map in greater relief.

(OOD) generalization remains a challenge (Yogatama et al., 2019; Linzen, 2020); and, while recent large pretrained language models help, they fail to close this gap (Hendrycks et al., 2020). This urges a closer look at datasets, where not all examples might contribute equally towards learning (Vodrahalli et al., 2018). However, the scale of data can make this assessment challenging. How can we automatically characterize data instances with respect to their role in achieving good performance in- and out-of- distribution? Answering this question may take us a step closer to bridging the gap between dataset collection and broader task 
objectives.

Drawing analogies from cartography, we propose to find coordinates for instances within the broader trends of a dataset. We introduce data maps: a model-based tool for contextualizing examples in a dataset. We construct coordinates for data maps by leveraging training dynamics - the behavior of a model as training progresses. We consider the mean and standard deviation of the gold label probabilities, predicted for each example across training epochs; these are referred to as confidence and variability, respectively $(\S 2)$.

Fig. 1 shows the data map for the SNLI dataset (Bowman et al., 2015) constructed using the RoBERTA-large model (Liu et al., 2019). The map reveals three distinct regions in the dataset: a region with instances whose true class probabilities fluctuate frequently during training (high variability), and are hence ambiguous for the model; a region with easy-to-learn instances that the model predicts correctly and consistently (high confidence, low variability); and a region with hard-to-learn instances with low confidence, low variability, many of which we find are mislabeled during annotation. ${ }^{1}$ Similar regions are observed across three other datasets: MultiNLI (Williams et al., 2018), WinoGrande (Sakaguchi et al., 2020) and SQuAD (Rajpurkar et al., 2016), with respect to respective ROBERTA-large classifiers.

We further investigate the above regions by training models exclusively on examples from each region ( $(3)$. Training on ambiguous instances promotes generalization to OOD test sets, with little or no effect on in-distribution (ID) performance. ${ }^{2}$ Our data maps also reveal that datasets contain a majority of easy-to-learn instances, which are not as critical for ID or OOD performance, but without any such instances, training could fail to converge (§4). In $\S 5$, we show that hard-to-learn instances frequently correspond to labeling errors. Lastly, we discuss connections between our measures and uncertainty measures ( $(6)$.

Our findings indicate that data maps could serve as effective tools to diagnose large datasets, at the reasonable cost of training a model on them. Locating different regions within the data might pave the way for constructing higher quality datasets., and ultimately models that generalize better. Our code

\footnotetext{
${ }^{1}$ All terms are defined with respect to the model.

${ }^{2}$ We define out-of-distribution (OOD) test sets as those which are collected independently of the original dataset, and ID test sets as those which are sampled from it.
}

and higher resolution visualizations are publicly available. $^{3}$

\section{Mapping Datasets with Training Dynamics}

Our goal is to construct Data Maps for datasets to help visualize a dataset with respect to a model, as well as understand the contributions of different groups of instances towards that model's learning. Intuitively, instances that a model always predicts correctly are different from those it almost never does, or those on which it vacillates. For building such maps, each instance in the dataset must be contextualized in the larger set. We consider one contextualization approach, based on statistics arising from the behavior of the training procedure across time, or the "training dynamics". We formally define our notations ( $(2.1)$ and describe our data maps (§2.2).

\subsection{Training Dynamics}

Consider a training dataset of size $N, \mathcal{D}=$ $\left\{\left(\boldsymbol{x}, y^{*}\right)_{i}\right\}_{i=1}^{N}$ where the $i$ th instance consists of the observation, $\boldsymbol{x}_{i}$ and its true label under the task, $y_{i}^{*}$. Our method assumes a particular model (family) whose parameters are selected to minimize empirical risk using a particular algorithm. ${ }^{4}$ We assume the model defines a probability distribution over labels given an observation. We assume a stochastic gradient-based optimization procedure is used, with training instances randomly ordered at each epoch, across $E$ epochs.

The training dynamics of instance $i$ are defined as statistics calculated across the $E$ epochs. The values of these measures then serve as coordinates in our map. The first measure aims to capture how confidently the learner assigns the true label to the observation, based on its probability distribution. We define confidence as the mean model probability of the true label $\left(y_{i}^{*}\right)$ across epochs:

$$
\hat{\mu}_{i}=\frac{1}{E} \sum_{e=1}^{E} p_{\boldsymbol{\theta}^{(e)}}\left(y_{i}^{*} \mid \boldsymbol{x}_{i}\right)
$$

where $p_{\boldsymbol{\theta}^{(e)}}$ denotes the model's probability with parameters $\boldsymbol{\theta}^{(e)}$ at the end of the $e^{\text {th }}$ epoch. ${ }^{5}$ In

\footnotetext{
${ }^{3}$ https://github.com/allenai/ cartography

${ }^{4}$ In this paper, the model is RoBERTA (Liu et al., 2019), currently established as a strong performer across many tasks.

${ }^{5}$ Note that $\hat{\mu}_{i}$ is with respect to the true label $y_{i}^{*}$, not the probability assigned to the model's highest-scoring label (as used in active learning, for example).
} 

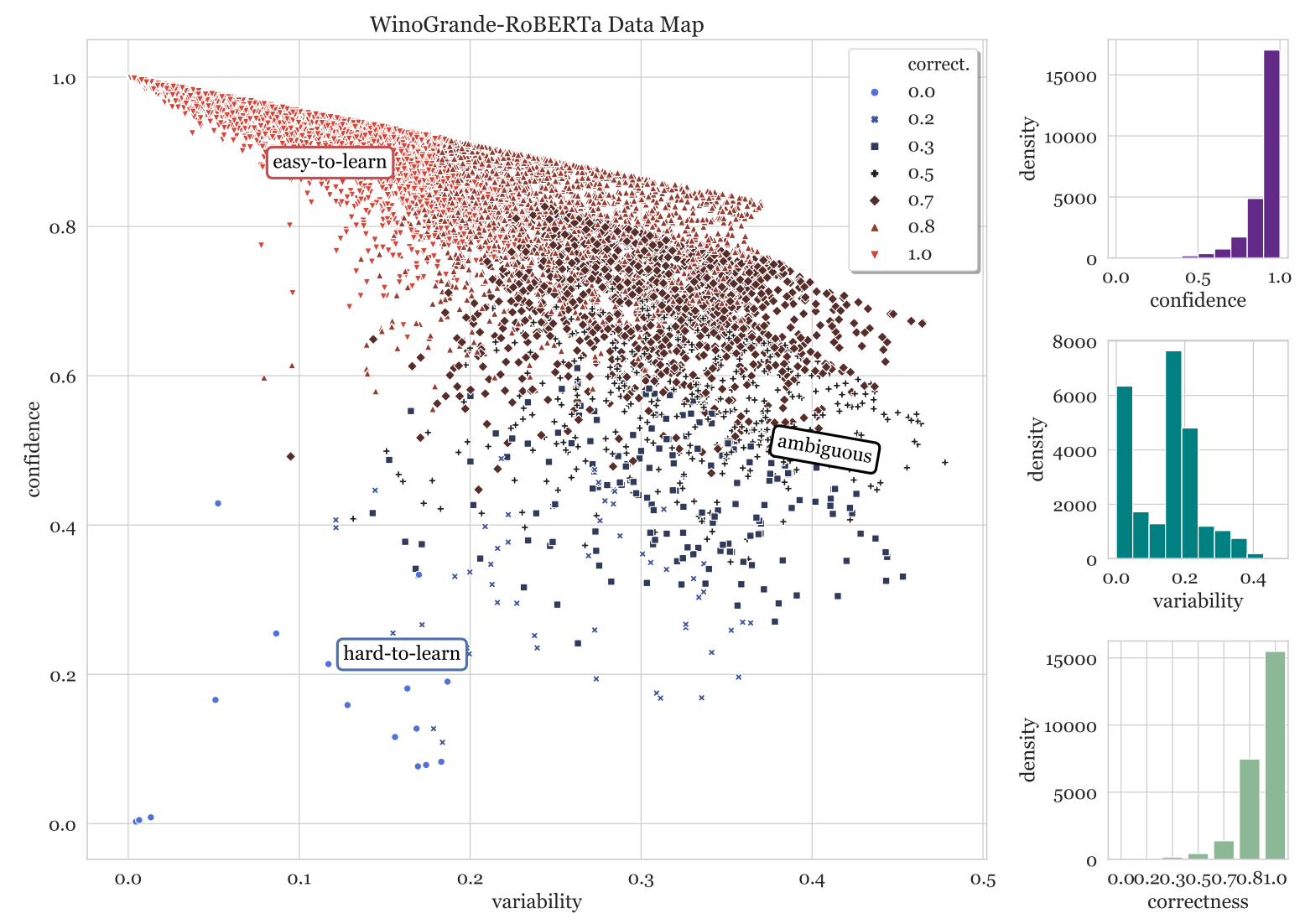

Figure 2: Data map for the WinoGrande (Sakaguchi et al., 2020) train set, based on a RoBERTA-large classifier, with the same axes as Fig. 1. Density plots for the three different measures based on training dynamics are shown towards the right. Hard-to-learn regions have lower density in WinoGrande, compared to SNLI, perhaps as a result of a rigorous validation of collected annotations. However, manual errors remain, which we showcase in Tab. 1 as well as in Section $\S 5$. The plot shows only $25 \mathrm{~K}$ train examples for clarity, and is best viewed enlarged.

some cases we also consider a coarser, and perhaps more intuitive statistic, the fraction of times the model correctly labels $\boldsymbol{x}_{i}$ across epochs, named correctness; this score only has $1+E$ possible values. Intuitively, a high-confidence instance is "easier" for the given learner.

Lastly, we also consider variability, which measures the spread of $p_{\boldsymbol{\theta}^{(e)}}\left(y_{i}^{*} \mid \boldsymbol{x}_{i}\right)$ across epochs, using the standard deviation:

$$
\hat{\sigma}_{i}=\sqrt{\frac{\sum_{e=1}^{E}\left(p_{\boldsymbol{\theta}^{(e)}}\left(y_{i}^{*} \mid \boldsymbol{x}_{i}\right)-\hat{\mu}_{i}\right)^{2}}{E}}
$$

Note that variability also depends on the gold label, $y_{i}^{*}$. A given instance to which the model assigns the same label consistently (whether accurately or not) will have low variability; one which the model is indecisive about across training, will have high variability.

Finally, we observe that confidence and variability are fairly stable across different pa- rameter initializations. ${ }^{6}$ Training dynamics can be computed at different granularities, such as steps vs. epochs; see App. A.1.

\subsection{Data Maps}

We construct data maps for four large datasets: WinoGrande (Sakaguchi et al., 2020)—a clozestyle task for commonsense reasoning, two NLI datasets (SNLI; Bowman et al., 2015; and MultiNLI; Williams et al., 2018), and QNLI, which is a sentence-level question answering task derived from SQuAD (Rajpurkar et al., 2016). All data maps are built with models based on RoBERTAlarge architectures. Details on the model and datasets can be found in App. $\S$ A.2 and $\S$ A.3.

Fig. 1 presents the data map for the $S N L I$ dataset. As is evident, the data follows a bell-shaped curve with respect to confidence and variability;

\footnotetext{
${ }^{6}$ The average Pearson correlation coefficient between five random seeds' resulting training runs is 0.75 or higher (for both measures, on WinoGrande).
} 


\begin{tabular}{|c|c|c|c|}
\hline & Instance & Option1 & Option2 \\
\hline \multirow{2}{*}{ 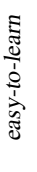 } & $\begin{array}{l}\text { The man chose to buy the roses in- } \\
\text { stead of the carnations because the -- } \\
\text { were more beautiful. }\end{array}$ & roses* & carnations \\
\hline & $\begin{array}{l}\text { We enjoyed the meeting tonight but } \\
\text { not the play as the _- was rather dull. }\end{array}$ & meeting & play* \\
\hline \multirow{3}{*}{ 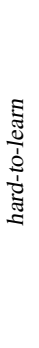 } & $\begin{array}{l}\text { Jason got into a deep financial hole, } \\
\text { unlike Joel, because _- managed their } \\
\text { fortune poorly. }\end{array}$ & Jason $^{+}$ & Joel* \\
\hline & $\begin{array}{l}\text { In the mornings, Aaron can hit the } \\
\text { snooze button a lot, and Samuel } \\
\text { can't. _- has to be at work at } 10 \text { am. }\end{array}$ & Aaron* & Samuel $^{-}$ \\
\hline & $\begin{array}{l}\text { Amy's handwriting was meticulous, } \\
\text { while Cynthia's handwriting was of- } \\
\text { ten sloppy, because -- was careless } \\
\text { about their work. }\end{array}$ & Amy* & Cynthia $^{+}$ \\
\hline \multirow{2}{*}{ 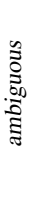 } & $\begin{array}{l}\text { The dog ran up to Leslie and away } \\
\text { from Lawrence because _- had soap } \\
\text { for the dog to take a bath. }\end{array}$ & Leslie $^{-}$ & Lawrence* \\
\hline & $\begin{array}{l}\text { Kayla dated many more people at } \\
\text { once than Betty, because _- was in an } \\
\text { exclusive relationship. }\end{array}$ & Kayla* & Betty $^{+}$ \\
\hline
\end{tabular}

Table 1: Examples from the WinoGrande train set from different regions in the data map, with gold standard*

labels. Our best assessment of the correct $^{+}$and equally plausible ${ }^{-}$labels are highlighted.

correctness further determines discrete regions therein. The vast majority of instances belong to the high confidence and low variability region of the map (Fig. 1, top-left). The model consistently predicts such instances correctly with high confidence; thus, we refer to them as easy-tolearn (for the model). A second, smaller group is formed by instances with low variability and low confidence (Fig. 1, bottom-left corner). Since such instances are seldom predicted correctly during training, we refer to them as hard-to-learn (for the model). The third notable group contains ambiguous examples, or those with high variability (Fig. 1, right-hand side); the model tends to be indecisive about these instances, such that they may or may not correspond to high confidence or correctness. We refer to such instances as ambiguous (to the model).

Fig. 2 shows the data map for WinoGrande, which exhibits high structural similarity to the SNLI data map (Fig. 1). The most remarkable difference between the maps is in the density of the hard-to-learn region, which is much lower for WinoGrande, as is evident from the histograms below. One explanation for this might be that WinoGrande labels were rigorously validated post annotation. App. $\S \mathrm{C}$ includes data maps for all four datasets, with respect to RoBERTA-large, in greater relief.

Different model architectures trained on a given dataset could be effectively compared using data maps, as an alternative to standard quantitative evaluation methods. App. $\S \mathrm{C}$ includes data maps for WinoGrande (Fig. 9b) and SNLI (Fig. 10 and Fig. 11) based on other (somewhat weaker) architectures. While data maps based on similar architectures have similar appearance, the regions to which a given instance belongs might vary. Data maps for weaker architectures still display similar regions, but the regions are not as distinct as those in ROBERTA based data maps.

Tab. 1 shows examples from WinoGrande belonging to the different regions defined above. easy-to-learn examples are straightforward for the model, as well as for humans. In contrast, most hard-to-learn and some ambiguous examples could be challenging for humans (see green highlights in Tab. 1), which might explain why the model shows lower confidence on them. These categories could be harder for models either because of labeling errors (blue highlights) or simply because the model is indecisive about the correct label. See App. §A.4 for similar examples from SNLI.

The next four sections include a diagnosis of the different data regions defined above. The effect of training models on each region on both in- and outof-distribution performance is studied in $\S 3$. The effect of selecting decreasing amounts of data is discussed in $\S 4$. We investigate the presence of mislabeled instances in the hard-to-learn regions of the data maps in $\S 5$. Lastly, we demonstrate connections between training dynamics measures and measures of uncertainty in $\S 6$.

\section{Data Selection using Data Maps}

Data maps reveal distinct regions in datasets; it is natural to wonder what roles do instances from different regions play in learning and generalization. We answer this empirically by training models exclusively on instances selected from distinct regions, followed by standard in-distribution (ID), as well as out-of-distribution (OOD) evaluation.

Our strategy is straightforward-we train the model from scratch on a subset of the training data selected by ranking instances based on the different training dynamics measures. ${ }^{7}$ We hypothesize that ambiguous and hard-to-learn regions could

\footnotetext{
${ }^{7}$ Hyperparameters are also tuned from scratch (App. §A.3).
} 


\begin{tabular}{|c|c|c|c|}
\hline & & WINOG. Val. (ID) & WSC (OOD) \\
\hline & $100 \%$ train & $79.7_{0.2}$ & $86.0_{0.1}$ \\
\hline & random & $73.3_{1.3}$ & $85.6_{0.4}$ \\
\hline & high-correctness & $70.8_{0.6}$ & $84.1_{0.4}$ \\
\hline & high-confidence & $69.4_{0.5}$ & $83.9_{0.5}$ \\
\hline$\Xi$ & low-variability & $70.1_{1.0}$ & $83.7_{1.4}$ \\
\hline & forgetting & $75.5_{1.3}$ & $84.8_{0.7}$ \\
\hline$m$ & AL-uncertainty & $75.7_{0.8}$ & $85.7_{0.8}$ \\
\hline & AL-greedyK & $74.2_{0.4}$ & $86.5_{0.5}$ \\
\hline & AFLite & $76.8_{0.8}$ & $86.6_{0.6}$ \\
\hline & low-correctness & $78.2_{0.6}$ & $86.3_{0.6}$ \\
\hline & hard-to-learn & $77.9_{1.3}$ & $87.2_{0.7}$ \\
\hline & ambiguous & $\mathbf{7 8 . 7} 7_{0.4}$ & $\mathbf{8 7 . 6}_{0.6}$ \\
\hline
\end{tabular}

Table 2: ID and OOD accuracies for RoBERTA-large models trained on different selections of WinoGrande. Reported values are averaged over 3 random seeds, with s.d. reported as a subscript. Selection of $33 \%$ training instances with highest variability (ambiguous) achieves the best OOD performance, outperforming all other baselines from this work, as well as prior work.

be the most informative for learning, since these examples are the most challenging for the model (Shrivastava et al., 2016). We compare these two settings to RoBERTA-large models trained on data subsets, selected using several other methods. All subsets considered contain $33 \%$ of the training data (to control for the effect of train data size on performance).

Baselines The two most natural baselines are those where all data is used (100\% train), and where a $33 \%$ random sample is used (random). Our second set of baselines considers subsets which are the most easy-to-learn for the model (highconfidence), and those that the model is most decisive about (low-variability), which comprises a mixture of easy-to-learn and hard-to-learn examples. We also consider baselines based on highand low-correctness. Finally, we also compare with our implementation of the following methods for data selection from prior work (discussed in $\S 7$ ): forgetting (Toneva et al., 2018), AFLite (LeBras et al., 2020), AL-uncertainty (Joshi et al., 2009), and AL-greedyK (Sener and Savarese, 2018).

Results We test our selections on the same datasets from the previous section-WinoGrande, SNLI, MultiNLI and QNLI. We report ID validation performance, and OOD performance on test sets either created independently of the dataset (NLI Diagnostics (Wang et al., 2019) for SNLI and MultiNLI, and WSC (Levesque et al., 2011) for
WinoGrande), or specifically to be adversarial to the dataset (Adversarial SQuAD (Jia and Liang, 2017) for QNLI); see App. §A.2 for details.

Tab. 2 shows our results on WinoGrande. ${ }^{8}$ Training on the most ambiguous data results in the best OOD performance, exceeding that of $100 \%$ train, even with just a third of the data. A similar effect is seen with hard-to-learn, as well as its coarsegrained counterpart, low-correctness. In each of the three cases, ID performance is also higher than all other 33\% baselines, though we observe some degradation compared to the full training set; this is expected as with larger amounts of data models tend to fit the dataset distribution rather than the task (Torralba and Efros, 2011). The only selection methods that underperform the random baseline are forgetting, and the ones where we select data the model is highly confident and decisive about (high-confidence, high-correctness, and lowvariability). The latter pattern, as well as our overall results, highlight the important role played by examples which are challenging for the model, i.e., ambiguous and hard-to-learn examples.

Given that our selection methods outperform baselines from prior work, we only report random and $100 \%$ train selection baselines on the remaining datasets, where we see similar trends. Tab. 3 shows results for $S N L I$ and MultiNLI, where the random selection baseline is already within $1 \%$ of the $100 \%$ train baseline. ${ }^{9}$ Selecting $33 \%$ of the most ambiguous examples achieves even better ID performance, within $0.2 \%$ of the $100 \%$ train baseline, while exceeding OOD performance substantially on each of the linguistic categories in the NLI Diagnostics test set. ${ }^{10}$ While hard-to-learn does not perform as well as ambiguous on most cases, it still matches or outperforms the $100 \%$ train baseline on OOD test sets. Tab. 4 shows a similar trend for QNLI, where we gain over 2\% performance on the OOD Adversarial SQuAD test set, with minimal loss in ID accuracy.

Overall, regions revealed by data maps provide ways to substantially improve OOD performance

\footnotetext{
${ }^{8}$ The official test set for WinoGrande has been filtered with AFLite, making ID evaluation more challenging than OOD. However, we apply all our selection methods (including the AFLite selection) on WinoGrande's unfiltered training data.

${ }^{9}$ The ID performance of all models exceeds human accuracy $(88 \%)$ for $S N L I$. However, the difference in ID and OOD performance in SNLI is quite high, showing that there is still room for improvement in the NLI task.

${ }^{10}$ While MultiNLI-mismatched is technically out-ofdomain, performance is close to matched (ID).
} 


\begin{tabular}{|c|c|c|c|c|c|c|c|c|c|c|c|c|c|c|}
\hline & & \multicolumn{6}{|c|}{$S N L I$} & \multicolumn{7}{|c|}{ MultiNLI } \\
\hline & & \multirow{2}{*}{$\frac{\text { ID }}{\text { Test }}$} & \multicolumn{5}{|c|}{ NLI Diagnostics (OOD) } & \multicolumn{2}{|c|}{ ID (Val.) } & \multicolumn{5}{|c|}{ NLI Diagnostics (OOD) } \\
\hline & & & Lex. & PAS & LS & Kno. & All & Mat. & MisM. & Lex. & PAS & LS & Kno. & All \\
\hline & $100 \%$ train & 92.0 & 54.6 & 67.9 & 62.7 & 52.1 & 61.8 & 90.2 & 90.1 & 59.9 & 68.4 & 67.3 & 57.8 & 65.0 \\
\hline \multirow{3}{*}{ 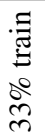 } & random & 91.3 & 53.0 & 66.8 & 59.7 & 50.7 & 60.4 & 89.8 & 89.2 & 59.3 & 69.6 & 66.5 & 56.3 & 64.6 \\
\hline & hard-to-learn & 91.8 & 55.2 & 69.1 & 63.2 & 51.7 & 62.0 & 89.5 & 89.7 & 59.3 & 68.9 & 69.5 & 58.8 & 65.3 \\
\hline & ambiguous & 92.2 & 58.5 & 67.9 & 64.1 & 54.2 & 63.5 & 90.1 & 89.3 & 63.5 & 71.0 & 68.9 & 59.2 & 66.9 \\
\hline
\end{tabular}

Table 3: ID and OOD accuracies for RoBERTA-large models trained on different selections of SNLI and MultiNLI; we report the best performance over 3 random seeds (see Appendix $\S$ B for SNLI validation results). ambiguous and hard-to-learn subsets of data promote OOD generalization, at minimal degradation of ID performance. OOD performance improves across all fine-grained linguistic categories in the NLI Diagnostics set.
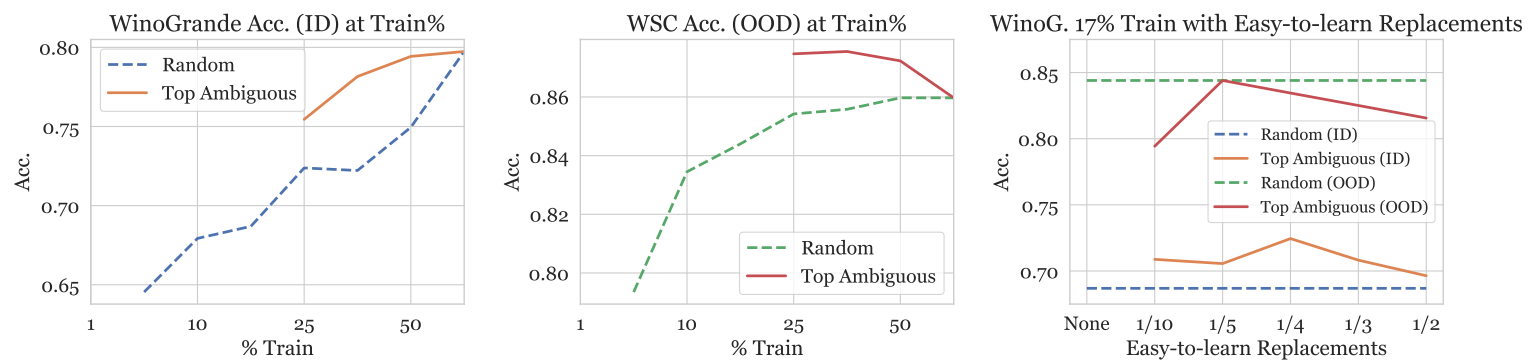

Figure 3: ID (left) and OOD (centre) WinoGrande performance with increasing \% of ambiguous (and randomlysampled) training data. RoBERTA-large optimization fails when trained on small amounts $(<25 \%)$ of the most ambiguous data (results correspond to majority baseline performance and are not shown here, for better visibility). (Right) Replacing small amounts of ambiguous examples from the 17\% subset with easy-to-learn examples results in successful optimization and ID improvements, at the cost of decreased OOD accuracy. All reported performances are averaged over 3 random seeds.

\begin{tabular}{|c|c|c|c|}
\hline & \multirow{2}{*}{$\frac{\text { In-dist. }}{\text { QNLI Val. }}$} & \multirow{2}{*}{$\frac{\text { Out-of-dist. }}{\text { Adversarial } S Q u A D}$} \\
\hline & & & \\
\hline & $100 \%$ train & $93.7_{0.3}$ & $81.7_{0.6}$ \\
\hline \multirow{2}{*}{ 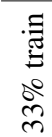 } & random & $92.7_{0.3}$ & $78.3_{0.4}$ \\
\hline & $\begin{array}{l}\text { hard-to-learn } \\
\text { ambiguous }\end{array}$ & $\begin{array}{l}93.3_{0.2} \\
93.8_{0.3}\end{array}$ & $\begin{array}{l}83.3_{0.6} \\
\text { 83.9 }{ }_{0.2}\end{array}$ \\
\hline
\end{tabular}

Table 4: QNLI performance on ID validation and OOD test sets, showing substantial improvements in the latter with a third of the original data. Reported values are averaged over 3 random seeds, with s.d. as subscripts.

across datasets. Regional selections of data not only improve model generalization, but also do so using substantially less data, providing a method to potentially speed up training. We note, however, that discovering such examples requires computing training dynamics, which involves training a model on the full dataset. Future directions involve building more efficient data maps, to better fulfill the training speedup potential.

\section{Role of Easy-to-Learn Instances}

Data maps uncover ambiguous regions, small subsets from which lead to improved OOD performance, with minimal degradation of ID performance ( $(3)$. We next investigate how performance is affected as we vary the size of the ambiguous subsets. We retrain our model with subsets containing the top 50\%, 33\%, 25\%, 17\%, 10\%, 5\% and $1 \%$ ambiguous instances of WinoGrande (Fig. 3, left and center). Large ambiguous subsets (25\% or more) result in high ID and OOD performance. Surprisingly however, for smaller ambiguous subsets (17\% or less), the model performs at chance level, despite random restarts. ${ }^{11}$ In contrast, a baseline that randomly selects subsets of similar sizes is able to learn (while naturally performing worse as data decreases, eventually failing at $1 \%$ ). This indicates that ambiguous instances alone might be insufficient for learning.

Given that the model barely struggles with easy-

\footnotetext{
${ }^{11}$ This is common for large models trained on small datasets (Devlin et al., 2019; Phang et al., 2018; Dodge et al., 2020).
} 

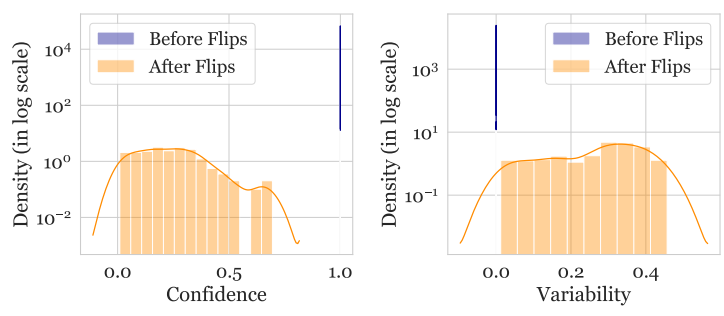

Figure 4: Retraining WinoGrande with $1 \%$ noised (label-flipped) data changes the training dynamics of the noisy examples. After retraining, there is a noticeable distributional shift towards lower confidence, with some shift towards higher variability as well.

to-learn instances (by definition), we next replace some ambiguous examples with easy-to-learn examples in the $17 \%$ most ambiguous subset. Interestingly, replacing just a tenth of the ambiguous data with easy-to-learn instances, the model not only successfully learns, but also outperforms the random selection baseline's ID performance (Fig. 3 right). This indicates that for successful optimization, it is important to include easier-tolearn instances. However, with too many replacements, performance starts decreasing again; this trend was seen in the previous section with the high-confidence baseline (Tab. 2). OOD performance shows a similar trend, but matches or is worse than the baseline. Selection of the optimal balance of easy-to-learn and ambiguous examples in low data regimes is an open problem; we defer this exploration to future work.

\section{Detecting Mislabeled Examples}

Crowdsourced datasets are often subject to noise attributed to incorrect labeled annotations (Sheng et al., 2008; Krishna et al., 2016; Ekambaram et al., 2017), which may lead to training models that are not representative of the task at hand. Recent studies have shown that over-parameterized neural networks can fit the incorrect labels blindly (Zhang et al., 2017), which might hurt their generalization ability (Hu et al., 2020). For large datasets, identifying mislabeled examples can be prohibitively expensive. Our data maps provide a semi-automated method to identify such mislabeled instances, without significantly more effort than simply training a model on the data. We hypothesize that hardto-learn examples - those with low confidencemight be mislabeled, as has also been suggested in prior work (Manning, 2011; Toneva et al., 2018).
To verify this hypothesis, we design an experimental setting where we artificially inject noise in the training data, by flipping the labels of $1 \%$ of the training data for WinoGrande. Motivated by our qualitative analysis (Tab 1), we select the candidates for flipping from the easy-to-learn regionthis minimizes the risk of selecting already mislabeled examples. We retrain RoBERTA with the partly noised data, and recompute confidence and variability of all instances. Fig. 4 shows the training dynamics measures, before and after re-training. Flipped instances move to the lower confidence regions after retraining, with some movement towards higher variability. This indicates that perhaps the hard-to-learn region (low confidence) of the map contains other mislabeled instances. We next explore a simple method to automatically detect such instances.

Automatic Noise Detection We train a linear model to classify examples as mislabeled (noise) or not, using a single feature: the confidence score from the retrained RoBERTA model on WinoGrande. This model is trained using a balanced training set for this task by sampling equal numbers of noisy (label-flipped) and clean examples from the original train set. This simple classifier is quite effective-a sanity check evaluation on a similarly constructed test set yields $100 \% \mathrm{~F} 1 .{ }^{12}$

Next, we run the trained noise classifier on the entire original training set, with features extracted from the original training dynamics measures (computed without added noise). We first observe that despite training on a balanced dataset, our classifier predicts only a few examples as mislabeled-only 31 WinoGrande instances (out a total of 40K). A similar experiment on $S N L I$ results in $15 \mathrm{~K}$ noisy examples (out of 500K). These results are encouraging and follow our intuitions that most instances in data are indeed labeled "correctly". Indeed, WinoGrande contains a lower portion of noisy examples, as indicated by our data maps (Fig. 2).

We further investigated these trends via a human evaluation on the output of the classifier. We created an evaluation set by randomly selecting 50 instances from each predicted class as per our classifier. Two of the authors re-annotated these 100 instances (without access to the original or predicted labels); some instances were annotated as too ambiguous for the authors. After discussions

\footnotetext{
${ }^{12} \mathrm{~A}$ similar experiment with only variability scores as features resulted in a much poorer classifier- $70 \% \mathrm{~F} 1$.
} 
to resolve their differences, both annotators agreed on $96 \%$ of the instances in each dataset. Using our annotations as a new gold standard, we found that for WinoGrande, $67 \%$ of the instances predicted as noisy by the linear classifier are indeed either mislabeled or ambiguous, compared to only $13 \%$ of the ones predicted as correctly labeled. Similar patterns are observed for SNLI (76\% vs. $4 \%$ ).

Our results demonstrate the potential of using data maps as a tool to "clean-up" datasets, by identifying mislabeled or ambiguous instances. ${ }^{13} \mathrm{No}$ tably, our results were obtained using a simple method; this encourages exploration of methods that might lead to more accurate noise-detectors.

\section{Training Dynamics as Uncertainty Measures}

We introduced data maps, and used training dynamics measures as coordinates for data points in $\S 2$. We now take a closer look at these measures, and find intuitive connections with measures of uncertainty. When a model fails to predict the correct label, the error may come from ambiguity inherent to the example (intrinsic uncertainty), but it may also come from the model's limitations (often referred to as model uncertainty). ${ }^{14}$ To understand how examples contribute to a dataset, it is important to separate these two sources of error.

We start by studying the relationship between intrinsic uncertainty and our training dynamics measures. Human agreement can serve as a proxy for intrinsic uncertainty. We estimate human agreement using the multiple human annotations available in SNLI's development set. ${ }^{15}$ For each annotator, we compute whether they agree with the majority label from the other four, breaking ties randomly and then averaging over annotators. ${ }^{16,17}$

Fig. 5 visualizes the relation between our training dynamics measures (confidence and variability) and human agreement, averaged over the examples. We observe a strong relationship be-

\footnotetext{
${ }^{13}$ In preliminary experiments, retraining WinoGrande after removal of noise did not yield a large difference in performance, given the relatively small amount of noise.

${ }^{14}$ These are also sometimes called the aleatoric and epistemic uncertainty, respectively (Gal, 2016).

${ }^{15}$ Only SNLI dev. and test set have multiple annotations on all instances. We obtain training dynamics with RoBERTAlarge run on train and dev. combined.

${ }^{16}$ Normally, this provides the minimum-variance unbiased estimate, though SNLI's development set throws away examples without a majority, which introduces some bias.

${ }^{17}$ Note that the model only has seen the majority vote, while we take into account all annotator labels to quantify agreement.
}

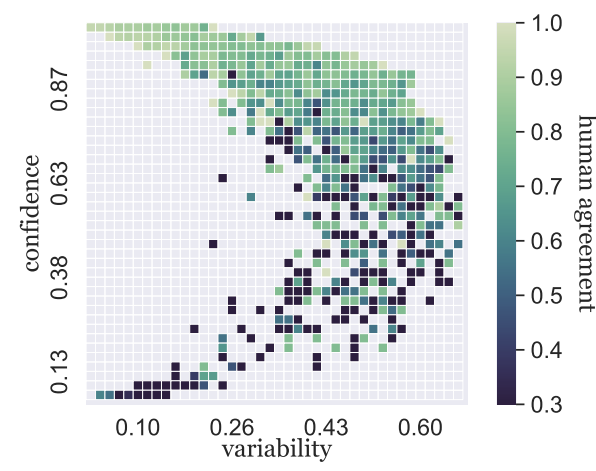

Figure 5: Visualizing human agreement on the SNLI (dev. set only) data map reveals its strong relationship to confidence. Each cell in the heatmap bins examples based on confidence and variability, then colors the cell by the mean human agreement.

tween human agreement and confidence: high confidence indicates high agreement between annotators, while low confidence often indicates disagreement on the example. In contrast, once confidence is known, variability does not provide much information about the agreement.

The connection between our second measure, variability, and model uncertainty is more straightforward: variability, by definition, captures exactly the uncertainty of the model. See App. B.1 for an additional discussion (with empirical justifications) on connections between training dynamics measures and dropout-based (Srivastava et al., 2014), first-principles uncertainty estimates.

These relations are further supported by previous work, which showed that deep ensembles provide well-calibrated uncertainty estimates (Lakshminarayanan et al., 2017; Gustafsson et al., 2019; Snoek et al., 2019). Generally, such approaches ensemble models trained from scratch; while ensembles of training checkpoints lose some diversity (Fort et al., 2019), they offer a cheaper alternative capturing some of the benefits (Chen et al., 2017a). Future work will involve investigation of such alternatives for building data maps.

\section{Related Work}

Our work builds data maps using training dynamics measures for scoring data instances. Loss landscapes (Xing et al., 2018) are similar to training dynamics, but also consider variables from the stochastic optimization algorithm. Toneva et al. (2018) also use training dynamics to find train examples which are frequently "forgotten", i.e., mis- 
classified during a later epoch of training, despite being classified correctly earlier; our correctness metric provides similar discrete scores, and results in models with better performance. Variants of such approaches address catastrophic forgetting, and are useful for analyzing data instances (Pan et al., 2020; Krymolowski, 2002).

Prior work has proposed other criteria to score instances. AFLite (LeBras et al., 2020) is an adversarial filtering algorithm which ranks instances based on their "predictability", i.e. the ability of simple linear classifiers to predict them correctly. While AFLite, among others ( $\mathrm{Li}$ and Vasconcelos, 2019; Gururangan et al., 2018), advocate removing "easy" instances from the dataset, our work shows that easy-to-learn instances can be useful. Similar intuitions have guided other work such as curriculum learning (Bengio et al., 2009) and self-paced learning (Kumar et al., 2010; Lee and Grauman, 2011) where all examples are prioritized based on their "difficulty".

Other approaches have used training loss (Han et al., 2018; Arazo et al., 2019; Shen and Sanghavi, 2019), confidence (Hovy et al., 2013), and meta-learning (Ren et al., 2018), to differentiate instances within datasets. Perhaps our measures are the closest to those from Chang et al. (2017); they propose prediction variance and threshold closeness - which correspond to variability and confidence, respectively. ${ }^{18}$ However, they use these measures to reweight all instances, similar to sampling effective batches in online learning (Loshchilov and Hutter, 2016). Our work, instead, does a hard selection for the purpose of studying different groups within data.

Our methods are also reminiscent of active learning methods (Settles, 2009; Peris and Casacuberta, 2018; P.V.S and Meyer, 2019), such as uncertainty sampling (Lewis and Gale, 1994) which selects (unlabeled) data points, which a model trained on a small labeled subset, has least confidence in, or predicts as farthest (in vector space, based on cosine similarity) (Sener and Savarese, 2018; Wolf, 2011). Our approach uses labeled data for selection, similar to core-set selection approaches (Wei et al., 2013). Active learning approaches could be used in conjunction with data maps to create better datasets, similar to approaches proposed in Mishra et al. (2020). For instance, creating datasets

\footnotetext{
${ }^{18}$ They also consider confidence intervals; our preliminary experiments, with and without, yielded similar results.
}

with more ambiguous examples (with respect to a given model) could make it beneficial for OOD generalization.

Data error detection also involves instance scoring. Influence functions (Koh and Liang, 2017), forgetting events (Toneva et al., 2018), cross validation (Chen et al., 2019), Shapely values (Ghorbani and Zou, 2019), and the area-under-margin metric (Pleiss et al., 2020) have all been used to identify mislabeled examples. Some approaches avoid hard examples altogether (Bottou et al., 2005; Northcutt et al., 2017) to reduce fit to noisy data. Our use of training dynamics to locate mislabeled examples involves minimal additional effort beyond training a model on the dataset.

\section{Conclusion}

We presented data maps: an automatic method to visualize and diagnose large datasets using training dynamics. Our data maps for four different datasets reveal similar terrains in each dataset: groups of ambiguous instances useful for high performance, easy-to-learn instances which aid optimization, and hard-to-learn instances which often correspond to data errors. While our maps are based on ROBERTA-large, the methods to build them are model-agnostic (App. §C.1). Our work shows the effectiveness of simple training dynamics measures based on mean and standard deviation; exploration of more sophisticated measures to build data maps is an exciting future direction. Data maps not only help diagnose and make better use of existing datasets, but also hold potential for guiding the construction of new datasets. Moreover, data maps could facilitate comparison of different model architectures trained on a given dataset, resulting in alternative evaluation methodologies. Our implementation is publicly available to facilitate such efforts. ${ }^{19}$

\section{Acknowledgements}

This research was supported in part by DARPA under the MCS program through NIWC Pacific (N66001-19-2-4031) grant, and by the Allen Distinguished Investigator Award. We thank the anonymous reviewers, and our colleagues from AI 2 and UWNLP, especially Ana Marasović, and Suchin Gururangan, for their helpful feedback.

\footnotetext{
${ }^{19}$ https://github.com/allenai/ cartography
} 


\section{References}

Stanislaw Antol, Aishwarya Agrawal, Jiasen Lu, Margaret Mitchell, Dhruv Batra, C. Lawrence Zitnick, and Devi Parikh. 2015. VQA: Visual Question Answering. In $I C C V$.

Eric Arazo, Diego Ortego, Paul Albert, Noel O'Connor, and Kevin Mcguinness. 2019. Unsupervised label noise modeling and loss correction. In ICML, pages 312-321.

Yoshua Bengio, Jérôme Louradour, Ronan Collobert, and Jason Weston. 2009. Curriculum learning. In ICML.

Léon Bottou, Jason Weston, and Gökhan H Bakir. 2005. Breaking SVM complexity with Cross-Training. In NeurIPS, pages 81-88. MIT Press.

Samuel R. Bowman, Gabor Angeli, Christopher Potts, and Christopher D. Manning. 2015. A large annotated corpus for learning natural language inference. In Proceedings of the 2015 Conference on Empirical Methods in Natural Language Processing, pages 632-642, Lisbon, Portugal. Association for Computational Linguistics.

Haw-Shiuan Chang, Erik Learned-Miller, and Andrew McCallum. 2017. Active bias: Training more accurate neural networks by emphasizing high variance samples. In NeurIPS, pages 1002-1012.

Hugh Chen, Scott Lundberg, and Su-In Lee. 2017a. Checkpoint ensembles: Ensemble methods from a single training process. ArXiv:1710.03282.

Pengfei Chen, Benben Liao, Guangyong Chen, and Shengyu Zhang. 2019. Understanding and utilizing deep neural networks trained with noisy labels. In ICML, volume 97 of Proceedings of Machine Learning Research, pages 1062-1070. PMLR.

Qian Chen, Xiaodan Zhu, Zhen-Hua Ling, Si Wei, Hui Jiang, and Diana Inkpen. 2017b. Enhanced LSTM for natural language inference. In Proceedings of the 55th Annual Meeting of the Association for Computational Linguistics (Volume 1: Long Papers), pages 1657-1668, Vancouver, Canada. Association for Computational Linguistics.

Jacob Devlin, Ming-Wei Chang, Kenton Lee, and Kristina Toutanova. 2019. BERT: Pre-training of deep bidirectional transformers for language understanding. In NAACL.

Jesse Dodge, Suchin Gururangan, Dallas Card, Roy Schwartz, and Noah A. Smith. 2019. Show your work: Improved reporting of experimental results. In EMNLP.

Jesse Dodge, Gabriel Ilharco, Roy Schwartz, Ali Farhadi, Hannaneh Hajishirzi, and Noah A. Smith. 2020. Fine-tuning pretrained language models: Weight initializations, data orders, and early stopping. arXiv:2002.06305.
R. Ekambaram, D. B. Goldgof, and L. O. Hall. 2017. Finding label noise examples in large scale datasets. In $S M C$, pages 2420-2424.

Stanislav Fort, Huiyi $\mathrm{Hu}$, and Balaji Lakshminarayanan. 2019. Deep ensembles: A loss landscape perspective. ArXiv preprint arXiv:1912.02757.

Yarin Gal. 2016. Uncertainty in Deep Learning. Ph.D. thesis, University of Cambridge.

Yarin Gal and Zoubin Ghahramani. 2016. Dropout as a bayesian approximation: Representing model uncertainty in deep learning. In ICML, volume 48, pages 1050-1059. PMLR.

Amirata Ghorbani and James Zou. 2019. Data shapley: Equitable valuation of data for machine learning.

Suchin Gururangan, Swabha Swayamdipta, Omer Levy, Roy Schwartz, Samuel Bowman, and Noah A. Smith. 2018. Annotation artifacts in natural language inference data. In Proceedings of the 2018 Conference of the North American Chapter of the Association for Computational Linguistics: Human Language Technologies, Volume 2 (Short Papers), pages 107-112, New Orleans, Louisiana. Association for Computational Linguistics.

Fredrik K Gustafsson, Martin Danelljan, and Thomas B Schön. 2019. Evaluating scalable bayesian deep learning methods for robust computer vision. ArXiv preprint arXiv:1906.01620.

Bo Han, Quanming Yao, Xingrui Yu, Gang Niu, Miao Xu, Weihua $\mathrm{Hu}$, Ivor W. Tsang, and Masashi Sugiyama. 2018. Co-teaching: Robust training of deep neural networks with extremely noisy labels. In NeurIPS, pages 8536-8546.

Dan Hendrycks, Xiaoyuan Liu, Eric Wallace, Adam Dziedzic, Rishabh Krishnan, and Dawn Song. 2020. Pretrained transformers improve out-of-distribution robustness. ArXiv preprint arXiv:2004.06100.

Dirk Hovy, Taylor Berg-Kirkpatrick, Ashish Vaswani, and Eduard Hovy. 2013. Learning whom to trust with MACE. In Proceedings of the 2013 Conference of the North American Chapter of the Association for Computational Linguistics: Human Language Technologies, pages 1120-1130, Atlanta, Georgia. Association for Computational Linguistics.

Wei Hu, Zhiyuan Li, and Dingli Yu. 2020. Simple and effective regularization methods for training on noisily labeled data with generalization guarantee. In ICLR. OpenReview.net.

Robin Jia and Percy Liang. 2017. Adversarial examples for evaluating reading comprehension systems. In Proceedings of the 2017 Conference on Empirical Methods in Natural Language Processing, pages 2021-2031, Copenhagen, Denmark. Association for Computational Linguistics. 
Ajay J Joshi, Fatih Porikli, and Nikolaos Papanikolopoulos. 2009. Multi-class active learning for image classification. In CVPR, pages 23722379. IEEE.

Jared Kaplan, Sam McCandlish, Tom Henighan, Tom B. Brown, Benjamin Chess, Rewon Child, Scott Gray, Alec Radford, Jeffrey Wu, and Dario Amodei. 2020. Scaling laws for neural language models.

Diederik P. Kingma and Jimmy Ba. 2014 Adam: A method for stochastic optimization. ArXiv:1412.6980.

Pang Wei Koh and Percy Liang. 2017. Understanding black-box predictions via influence functions. In ICML, pages 1885-1894. JMLR. org.

Ranjay A. Krishna, Kenji Hata, Stephanie Chen, Joshua Kravitz, David A. Shamma, Fei-Fei Li, and Michael S. Bernstein. 2016. Embracing error to enable rapid crowdsourcing. In $\mathrm{CHI}$, pages $3167-$ 3179. ACM.

Yuval Krymolowski. 2002. Distinguishing easy and hard instances. In COLING.

M Pawan Kumar, Benjamin Packer, and Daphne Koller. 2010. Self-paced learning for latent variable models. In NeurIPS, pages 1189-1197.

Balaji Lakshminarayanan, Alexander Pritzel, and Charles Blundell. 2017. Simple and scalable predictive uncertainty estimation using deep ensembles. In NeurIPS, pages 6402-6413.

Ronan LeBras, Swabha Swayamdipta, Chandra Bhagavatula, Rowan Zellers, Matthew E. Peters, Ashish Sabharwal, and Yejin Choi. 2020. Adversarial filters of dataset biases. In ICML.

Yong Jae Lee and Kristen Grauman. 2011. Learning the easy things first: Self-paced visual category discovery. CVPR, pages 1721-1728.

Hector J Levesque, Ernest Davis, and Leora Morgenstern. 2011. The Winograd schema challenge. In $A A A I$, volume 46, page 47.

David D. Lewis and William A. Gale. 1994. A sequential algorithm for training text classifiers. In SIGIR, SIGIR '94, page 3-12, Berlin, Heidelberg. SpringerVerlag.

Yi Li and Nuno Vasconcelos. 2019. REPAIR: Removing representation bias by dataset resampling. IEEE.

Tal Linzen. 2020. How can we accelerate progress towards human-like linguistic generalization? In $A C L$.

Yinhan Liu, Myle Ott, Naman Goyal, Jingfei Du, Mandar S. Joshi, Danqi Chen, Omer Levy, Mike Lewis, Luke S. Zettlemoyer, and Veselin Stoyanov. 2019. RoBERTa: A robustly optimized BERT pretraining approach. ArXiv:1907.11692.
Ilya Loshchilov and Frank Hutter. 2016. Online batch selection for faster training of neural networks. In ICLR.

Christopher D. Manning. 2011. Part-of-speech tagging from $97 \%$ to $100 \%$ : Is it time for some linguistics? In CICLing, CICLing'11, page 171-189, Berlin, Heidelberg. Springer-Verlag.

Swaroop Mishra, Anjana Arunkumar, Bhavdeep Sachdeva, Chris Bryan, and Chitta Baral. 2020. DQI: Measuring Data Quality in NLP.

Curtis G. Northcutt, Tailin Wu, and Isaac L. Chuang. 2017. Learning with confident examples: Rank pruning for robust classification with noisy labels.

Pingbo Pan, Siddharth Swaroop, Alexander Immer, Runa Eschenhagen, Richard E. Turner, and Mohammad Emtiyaz Khan. 2020. Continual deep learning by functional regularisation of memorable past.

Álvaro Peris and Francisco Casacuberta. 2018. Active learning for interactive neural machine translation of data streams. In CoNLL, pages 151-160.

Jason Phang, Thibault Févry, and Samuel R. Bowman. 2018. Sentence encoders on STILTs: Supplementary training on intermediate labeled-data tasks. arXiv:1811.01088.

Geoff Pleiss, Tianyi Zhang, Ethan R. Elenberg, and Kilian Q. Weinberger. 2020. Identifying mislabeled data using the area under the margin ranking.

Avinesh P.V.S and Christian M. Meyer. 2019. Dataefficient neural text compression with interactive learning. In $N A A C L$, pages 2543-2554. Association for Computational Linguistics.

Pranav Rajpurkar, Jian Zhang, Konstantin Lopyrev, and Percy Liang. 2016. SQuAD: 100, 000+ questions for machine comprehension of text. In EMNLP.

Mengye Ren, Wenyuan Zeng, Bin Yang, and Raquel Urtasun. 2018. Learning to reweight examples for robust deep learning.

Olga Russakovsky, Jia Deng, Hao Su, Jonathan Krause, Sanjeev Satheesh, Sean Ma, Zhiheng Huang, Andrej Karpathy, Aditya Khosla, Michael Bernstein, Alexander C. Berg, and Li Fei-Fei. 2015. ImageNet Large Scale Visual Recognition Challenge. IJCV.

Keisuke Sakaguchi, Ronan Le Bras, Chandra Bhagavatula, and Yejin Choi. 2020. Winogrande: An adversarial winograd schema challenge at scale. In AAAI.

Ozan Sener and Silvio Savarese. 2018. Active learning for convolutional neural networks: A core-set approach. In ICLR.

Burr Settles. 2009. Active learning literature survey. Technical report, University of Wisconsin-Madison Department of Computer Sciences. 
Yanyao Shen and Sujay Sanghavi. 2019. Learning with bad training data via iterative trimmed loss minimization. In ICML, pages 5739-5748.

Victor S Sheng, Foster Provost, and Panagiotis G Ipeirotis. 2008. Get another label? improving data quality and data mining using multiple, noisy labelers. In SIGKDD, pages 614-622.

Abhinav Shrivastava, Abhinav Gupta, and Ross Girshick. 2016. Training region-based object detectors with online hard example mining. In $C V P R$, pages 761-769.

Jasper Snoek, Yaniv Ovadia, Emily Fertig, Balaji Lakshminarayanan, Sebastian Nowozin, D Sculley, Joshua Dillon, Jie Ren, and Zachary Nado. 2019. Can you trust your model's uncertainty? evaluating predictive uncertainty under dataset shift. In NeurIPS, pages 13969-13980.

Nitish Srivastava, Geoffrey Hinton, Alex Krizhevsky, Ilya Sutskever, and Ruslan Salakhutdinov. 2014 Dropout: a simple way to prevent neural networks from overfitting. The journal of machine learning research, 15(1):1929-1958.

Alon Talmor, Yanai Elazar, Yoav Goldberg, and Jonathan Berant. 2019. olmpics - on what language model pre-training captures. ArXiv:1912.13283.

Mariya Toneva, Alessandro Sordoni, Remi Tachet des Combes, Adam Trischler, Yoshua Bengio, and Geoffrey J Gordon. 2018. An empirical study of example forgetting during deep neural network learning. In ICLR.

Antonio Torralba and Alexei A Efros. 2011. Unbiased look at dataset bias. In CVPR 2011, pages 15211528. IEEE.

Kailas Vodrahalli, Ke Li, and Jitendra Malik. 2018. Are all training examples created equal? an empirical study. ArXiv:1811.12569.

Alex Wang, Yada Pruksachatkun, Nikita Nangia, Amanpreet Singh, Julian Michael, Felix Hill, Omer Levy, and Samuel R. Bowman. 2019. SuperGLUE: A stickier benchmark for general-purpose language understanding systems. In NeurIPS.

Alex Wang, Amanpreet Singh, Julian Michael, Felix Hill, Omer Levy, and Samuel Bowman. 2018. GLUE: A multi-task benchmark and analysis platform for natural language understanding. In Proceedings of the 2018 EMNLP Workshop BlackboxNLP: Analyzing and Interpreting Neural Networks for NLP, pages 353-355, Brussels, Belgium. Association for Computational Linguistics.

Kai Wei, Yuzong Liu, Katrin Kirchhoff, and Jeff Bilmes. 2013. Using document summarization techniques for speech data subset selection. In Proceedings of the 2013 Conference of the North American Chapter of the Association for Computational Linguistics: Human Language Technologies, pages 721-726.
Adina Williams, Nikita Nangia, and Samuel Bowman. 2018. A broad-coverage challenge corpus for sentence understanding through inference. In Proceedings of the 2018 Conference of the North American Chapter of the Association for Computational Linguistics: Human Language Technologies, Volume 1 (Long Papers), pages 1112-1122, New Orleans, Louisiana. Association for Computational Linguistics.

Gert W Wolf. 2011. Facility location: concepts, models, algorithms and case studies. series: Contributions to management science. IJGIS, 25(2):331333.

Thomas Wolf, Lysandre Debut, Victor Sanh, Julien Chaumond, Clement Delangue, Anthony Moi, Pierric Cistac, Tim Rault, R'emi Louf, Morgan Funtowicz, and Jamie Brew. 2019. Huggingface's transformers: State-of-the-art natural language processing. ArXiv:1910.03771.

Chen Xing, Devansh Arpit, Christos Tsirigotis, and Yoshua Bengio. 2018. A walk with SGD.

Dani Yogatama, Cyprien de Masson d'Autume, Jerome Connor, Tomas Kocisky, Mike Chrzanowski, Lingpeng Kong, Angeliki Lazaridou, Wang Ling, Lei Yu, Chris Dyer, et al. 2019. Learning and evaluating general linguistic intelligence. ArXiv preprint arXiv:1901.11373.

Chiyuan Zhang, Samy Bengio, Moritz Hardt, Benjamin Recht, and Oriol Vinyals. 2017. Understanding deep learning requires rethinking generalization. In ICLR. OpenReview.net. 


\section{A Supplemental Material}

\section{A.1 Training Dynamics Computation}

Both confidence and variability are computed across epochs, but could alternatively be computed over other granularities, e.g. over every few steps of optimization. This might enable more efficient computation of the same. However, care must be taken to ignore the first few steps till optimization stabilizes. In our experiments, we considered all epochs including the first to compute the training dynamics, since the first epoch contains multiple steps of optimization for large training sets.

Moreover, it is possible to stop training early, or before the training converges for computing training dynamics. This early burn-out scheme results in confidence and variability measures which correlate well with confidence and variability (see Fig. 6). For our experiments, we use later burn-outs corresponding to model convergence.

\section{A.2 Datasets}

This appendix provides further details on datasets. We perform our experimental evaluation on four large datasets, each with at least $10 \mathrm{~K}$ instances. Sizes of the different datasets are reported in Tab. 5. Instances in each of the original datasets are labeled by crowdworkers, whereas the OOD test sets are either manually or semi-automatically created. The performance in each case is reported as accuracy.

WinoGrande This dataset contains a large scale crowd-sourced collection of Winograd schema challenge (WSC Levesque et al., 2011) style questions. Commonsense reasoning is required to select an entity from a pair of entities to complete a sentence. Following Sakaguchi et al. (2020), we use the multiple choice architecture based on RoBERTA (Liu et al., 2019). For OOD evaluation, we use the validation set from the original WSC as provided under the SuperGLUE benchmark (Wang et al., 2019). We used a rule-based method to convert WSC validation and training data to the cloze-style format followed in WinoGrande, removing all the repetitions included in the training data. Figure 2 shows the data map for WinoGrande.

SNLI and MultiNLI The task of natural language inference involves prediction of the relationship between a premise and hypothesis sentence pair. The label determines whether the hypothesis entails, contradicts or is neutral to the premise. We

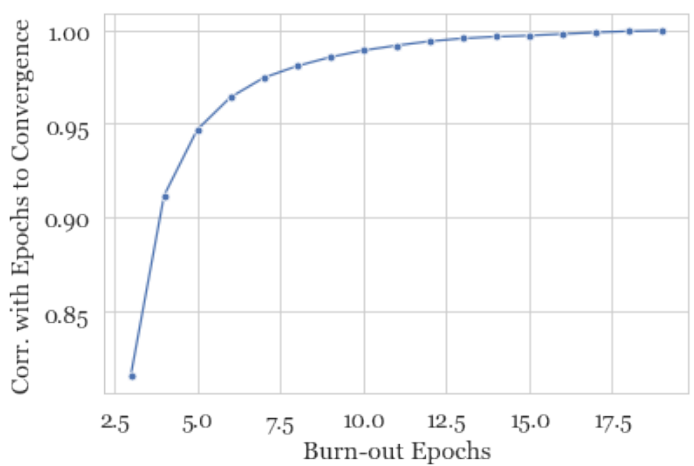

Figure 6: Pearson correlation coefficient of instance variability on WinoGrande between training dynamics when model is trained to convergence and when model is stopped early. The high correlation indicates that training till convergence is not required to compute a good approximation of the training dynamics.

experiment with the Stanford natural language inference (SNLI) dataset (Bowman et al., 2015) and its multi-genre counterpart, MultiNLI (Williams et al., 2018). ${ }^{20}$ Several challenge sets have been proposed to evaluate models OOD. As an OOD test set, we consider NLI diagnostics (Wang et al., 2018) which contains a set of hand-crafted examples designed to demonstrate NLI model performance on several fine-grained semantic categories, such as lexical semantics, logical reasoning, predicate argument structure and commonsense knowledge. In addition, we also report performance on the OOD mismatched MultiNLI validation set. Figure 8a shows the data map for MultiNLI.

QNLI Rajpurkar et al. (2016) proposed the $S Q u A D$ dataset containing question and document pairs, where the answer to the question is a span in the document. The QNLI dataset, provided as part of the GLUE benchmark (Wang et al., 2018) reformulates this as a sentence-level binary classification task. Here, the goal is to determine if a candidate sentence from the document contains the answer to the given question. As an OOD test set, we consider the Adversarial SQuAD challenge set (Jia and Liang, 2017) where distractor sentences are added to the document to confound the model. We automatically convert this to the QNLI format. Figure 9a shows the data map for QNLI.

\footnotetext{
${ }^{20}$ For MultiNLI, we use the version released under the GLUE benchmark (Wang et al., 2018).
} 


\begin{tabular}{lrrrrr}
\hline & \multicolumn{3}{c}{ In-dist. } & & Out-of-dist. \\
\cline { 2 - 3 } & Train & Val. & Test & & Test \\
\hline WinoGrande & 40399 & 1268 & - & & 424 \\
SNLI & 549368 & 9843 & 9825 & & 1105 \\
MultiNLI & 392703 & 9816 & 9833 & & 1105 \\
QNLI & 104744 & 5464 & - & & 5324 \\
\hline
\end{tabular}

Table 5: Dataset sizes. ID test set in MultiNLI is the mismatched validation set, which we did not use for validation, but as test. We did not use the provided test sets in WinoGrande and QNLI, rather report OOD performance for both cases.

\section{A.3 Experimental Settings}

For each of our classifiers, we minimize cross entropy with the Adam optimizer (Kingma and Ba, 2014) following the AdamW learning rate schedule from PyTorch ${ }^{21}$. Each experiment is run with 3 random seeds and a learning rate $^{22}$ chosen using the AllenTune package (Dodge et al., 2019). Initializations greatly affect performance, as noted in Dodge et al. (2020). WinoGrande and SNLI ROBERTA-large models are trained for 6 epochs, and MultiNLI and QNLI are trained for 5 epochs each. Each experiment is performed on a single Quadro RTX 8000 GPU. Based on the available GPU memory, our experiments on all datasets use a batch size of 96, except for WinoGrande, where a batch size of 64 is used. Our implementation uses the Huggingface Transformers library (Wolf et al., 2019). For the active learning baselines, we train a acquisition model using ROBERTA-large on a randomly sampled $1 \%$ subset of the full training set.

\section{A.4 SNLI Qualitative Analysis}

Qualitative samples from different regions of the SNLI data map are provided in Tab. 6.

\section{B Additional Results}

Results on the SNLI validation set are provided in Tab. 7.

\section{B.1 Training Dynamics vs. Dropout}

To empirically test the hypothesis that confidence and variability from the training dynamics respectively quantify intrinsic and model uncertainty, we compare confidence and variability against an established method of capturing intrinsic and

\footnotetext{
${ }^{21}$ pytorch.org

${ }^{22}$ Learning rate is chosen using a log-uniform sampling strategy from the range (5e-6, 2e-5).
}

model uncertainty from the literature based on dropout (Srivastava et al., 2014). Dropout can be seen as variational Bayesian inference $(\mathrm{Gal}$ and Ghahramani, 2016), with predictions from different dropout masks corresponding to predictions sampled from the posterior. Thus, confidence and variability computed from sampled dropout predictions measure the average and standard deviation of the gold label's probability under the posterior-quantifying the intrinsic and model uncertainty in a principled way.

We computed confidence and variability from both training dynamics and dropout on WinoGrande's development set. ${ }^{23}$ Figure 7 visualizes a regression analysis of the relationship between confidence and variability from training dynamics and dropout. confidence from training dynamics and dropout correlate between 0.450 and 0.452 for Pearson's $r$ at $95 \%$ confidence. Likewise, variability from training dynamics and dropout share a Pearson's $r$ from 0.390 to 0.393 at $95 \%$ confidence. Thus, the training dynamics empirically demonstrate a positive, predictive relationship with these first-principles estimates of the intrinsic and model uncertainty. Compared to dropout, however, training dynamics have the pragmatic advantage that all information required to calculate them is already available from training, without additional work or computation.

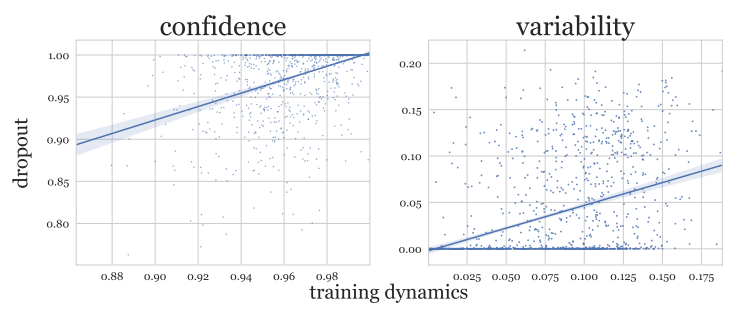

Figure 7: The confidence (left) and variability (right) from sampled dropout predictions correlate positively with those from the training dynamics on WinoGrande (dev. set). Shaded regions are bootstrapped 95\% confidence intervals for the regression line.

\section{Additional Data Maps}

All the data maps have been provided in Fig. 8 and Fig. 9.

\footnotetext{
${ }^{23}$ To compute training dynamics, we trained a model on the combined training and development sets for WinoGrande. In contrast, the dropout model was trained only on WinoGrande's training set then run on development, to avoid over-fitting and provide higher quality uncertainty estimates.
} 


\begin{tabular}{|c|c|c|c|c|}
\hline & Premise & Hypothesis & Gold Label & Our Assessment \\
\hline \multirow{2}{*}{ 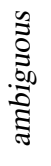 } & A mom is feeding two babies. & $\begin{array}{l}\text { A mom is giving her children } \\
\text { carrots to eat. }\end{array}$ & Contradiction $^{-}$ & Neutral \\
\hline & $\begin{array}{l}\text { Smiling woman in a blue apron standing in } \\
\text { front of a pile of bags and boxes. }\end{array}$ & $\begin{array}{l}\text { The woman is wearing a red } \\
\text { dress. }\end{array}$ & Neutral & \\
\hline \multirow{3}{*}{ 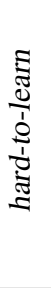 } & $\begin{array}{l}\text { Photographers take pictures of a girl sitting in } \\
\text { a street. }\end{array}$ & $\begin{array}{l}\text { The photographer is taking a pic- } \\
\text { ture of a boy. }\end{array}$ & Entailment $^{-}$ & Contradiction \\
\hline & $\begin{array}{l}\text { A group of men in a blue car driving on the } \\
\text { track. }\end{array}$ & $\begin{array}{l}\text { One woman is driving the blue } \\
\text { car. }\end{array}$ & Entailment $^{-}$ & Contradiction \\
\hline & $\begin{array}{l}\text { Pedestrians walking down the street passing } \\
\text { The Temple Bar. }\end{array}$ & The pedestrians are outside. & Contradiction $^{-}$ & Entailment \\
\hline \multirow{2}{*}{ 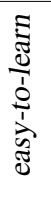 } & $\begin{array}{l}\text { Four musicians play their instruments on the } \\
\text { street while a young man on a bike stands by } \\
\text { to listen. }\end{array}$ & $\begin{array}{l}\text { a kid in a car goes through a } \\
\text { drive thru }\end{array}$ & Contradiction & \\
\hline & $\begin{array}{l}\text { A girl sits with excavating tools examining a } \\
\text { rock. }\end{array}$ & $\begin{array}{l}\text { Two men writing a draft of a } \\
\text { speech. }\end{array}$ & Contradiction & \\
\hline
\end{tabular}

Table 6: Examples from SNLI belonging to different regions in the data map. Cases where authors disagree with the gold standard are highlighted in blue ${ }^{-}$

\begin{tabular}{|c|c|c|}
\hline & & SNLI Val. (In-dist.) \\
\hline & $100 \%$ train & 93.1 \\
\hline$\Xi$ & random & 92.1 \\
\hline m & hard-to-learn & 92.6 \\
\hline
\end{tabular}

Table 7: SNLI validation performance comparing different selection methods. Reported numbers are the best of 3 runs across different seeds.

\section{C.1 Effect of Encoder in building Data Maps}

While training dynamics are inherently model dependent, data maps can be built for any model, and might reveal similar structures. Since models can be of varying capacities with respect to a task or dataset, instances might receive different co-ordinates on data maps built based on different models. For instance, BERT is known to be worse at reasoning than RoBERTA (Sakaguchi et al., 2020; Talmor et al., 2019), and RoBERTA being a larger model is likely very sample efficient (Kaplan et al., 2020). However, the overall structure of data maps based on different models remains the same; Fig. 9b shows the data map built for WinoGrande using a BERT-large classifier.

Four different architectures for the SNLI dataset are compared in Fig. 10 and Fig. 11. 

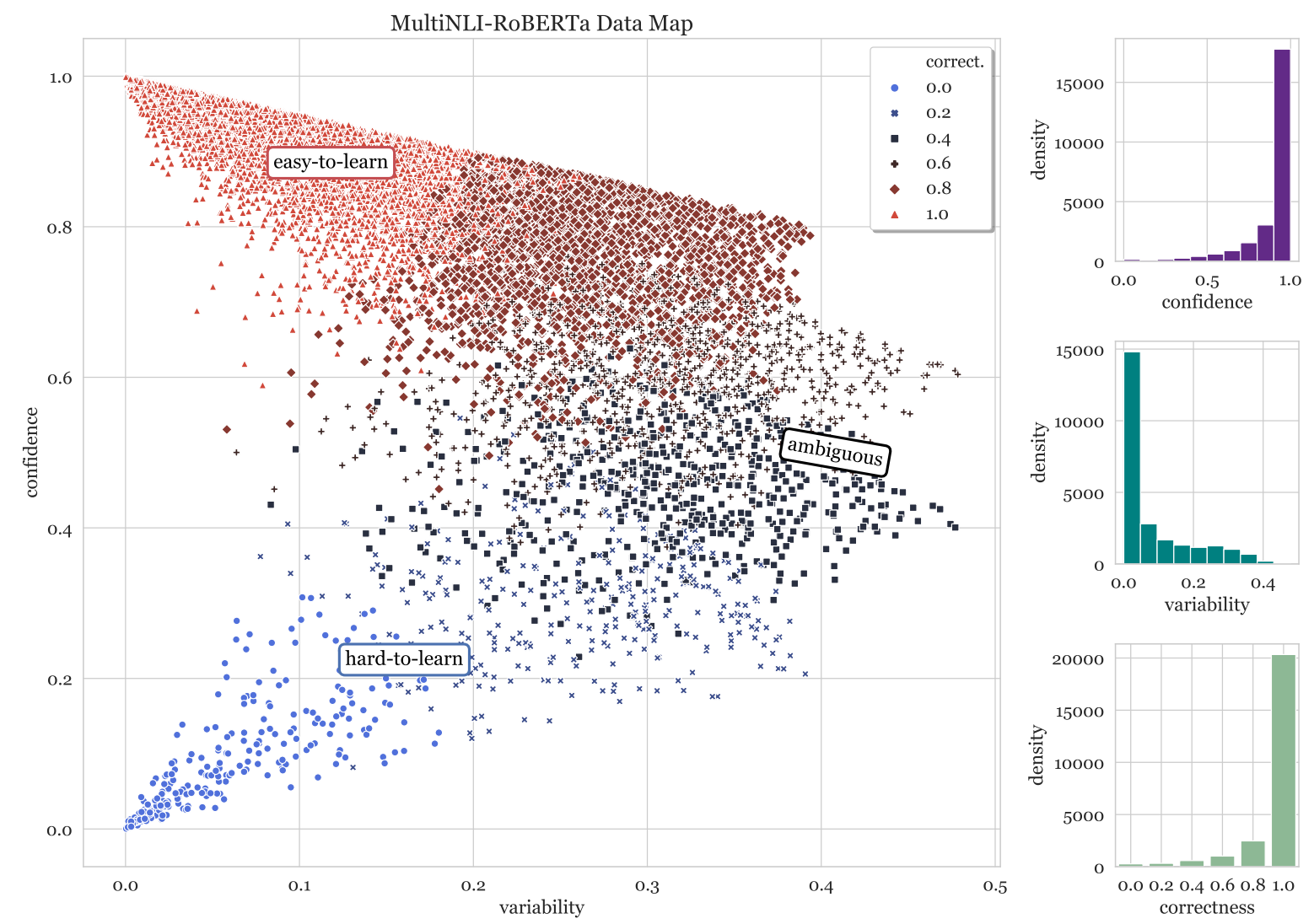

(a) Data Map for MultiNLI (Williams et al., 2018) and density plots for different measures based on training dynamics (below). For clarity we only use 50K random samples from MultiNLI in the scatter plot. Trends are very similar to SNLI, even though MultiNLI contains samples from diverse genres.
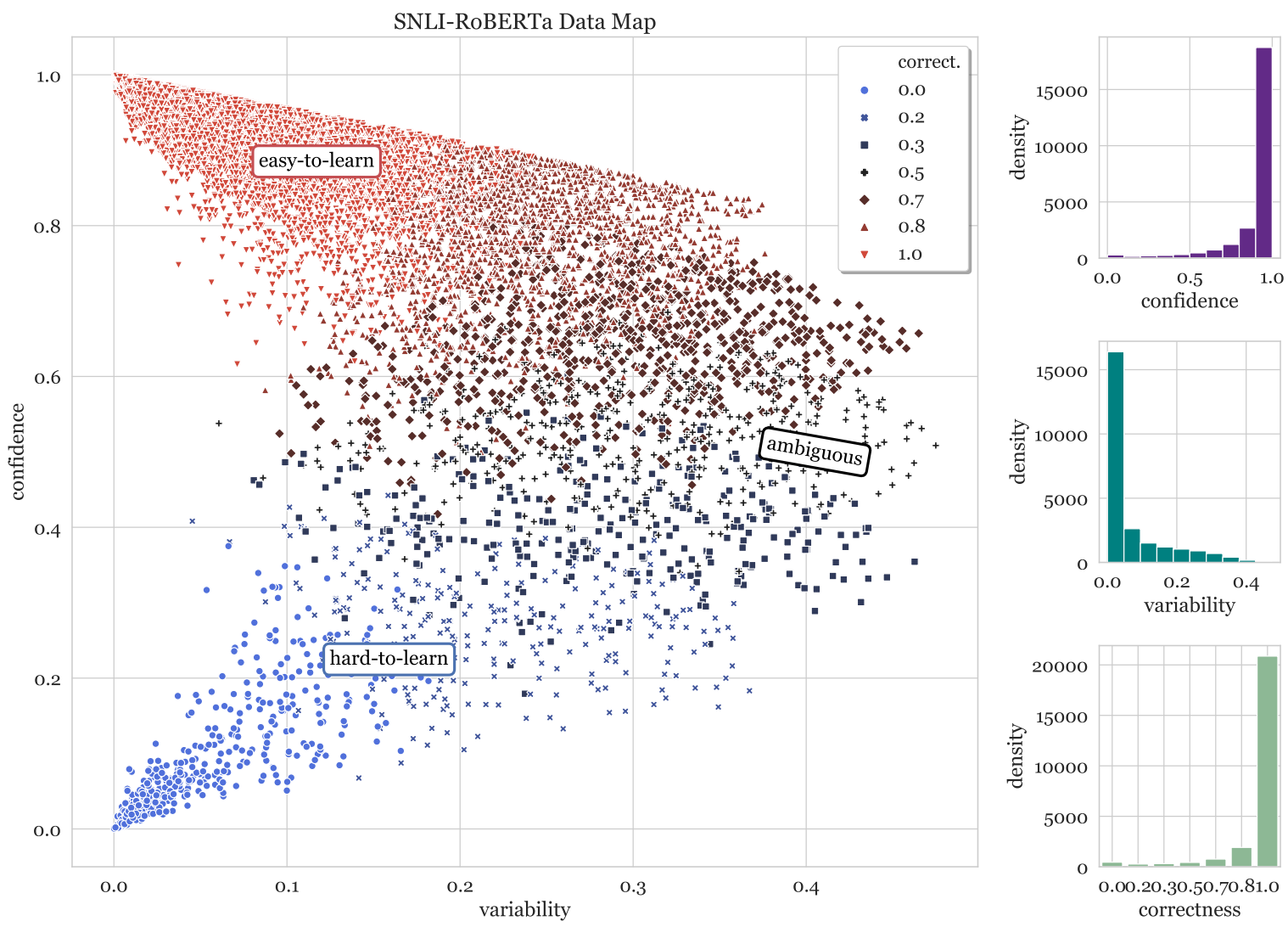

(b) (Left) Data Map for SNLI, same as Fig. 1, provided here in greater relief again for comparison with other datasets. SNLI is larger than all other datasets, and thus has a higher density of easy-to-learn examples. (Right) Densities of the above statistics across the entire dataset; examples which are easy-to-learn (for 920 BERTA) form the vast majority of SNLI.

Figure 8: Data maps for *NLI datasets; each data map plots $25 \mathrm{~K}$ instances, for clarity. 

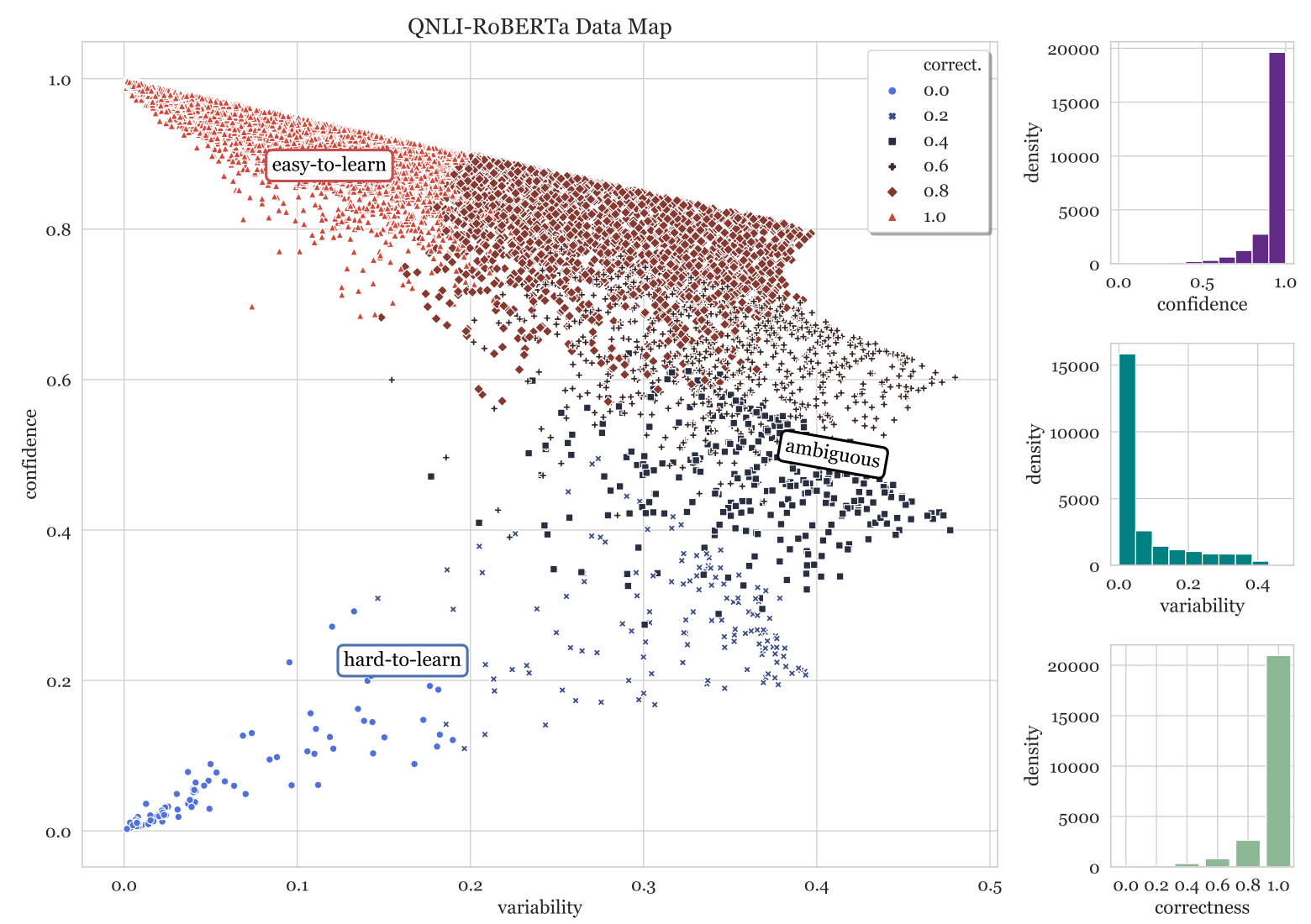

(a) Data map for the sentence-level SQuAD dataset, QNLI (left) and density plots for different measures based on training dynamics (right). Unlike other datasets, QNLI has fewer instances with low variability and confidence close to 0.5.
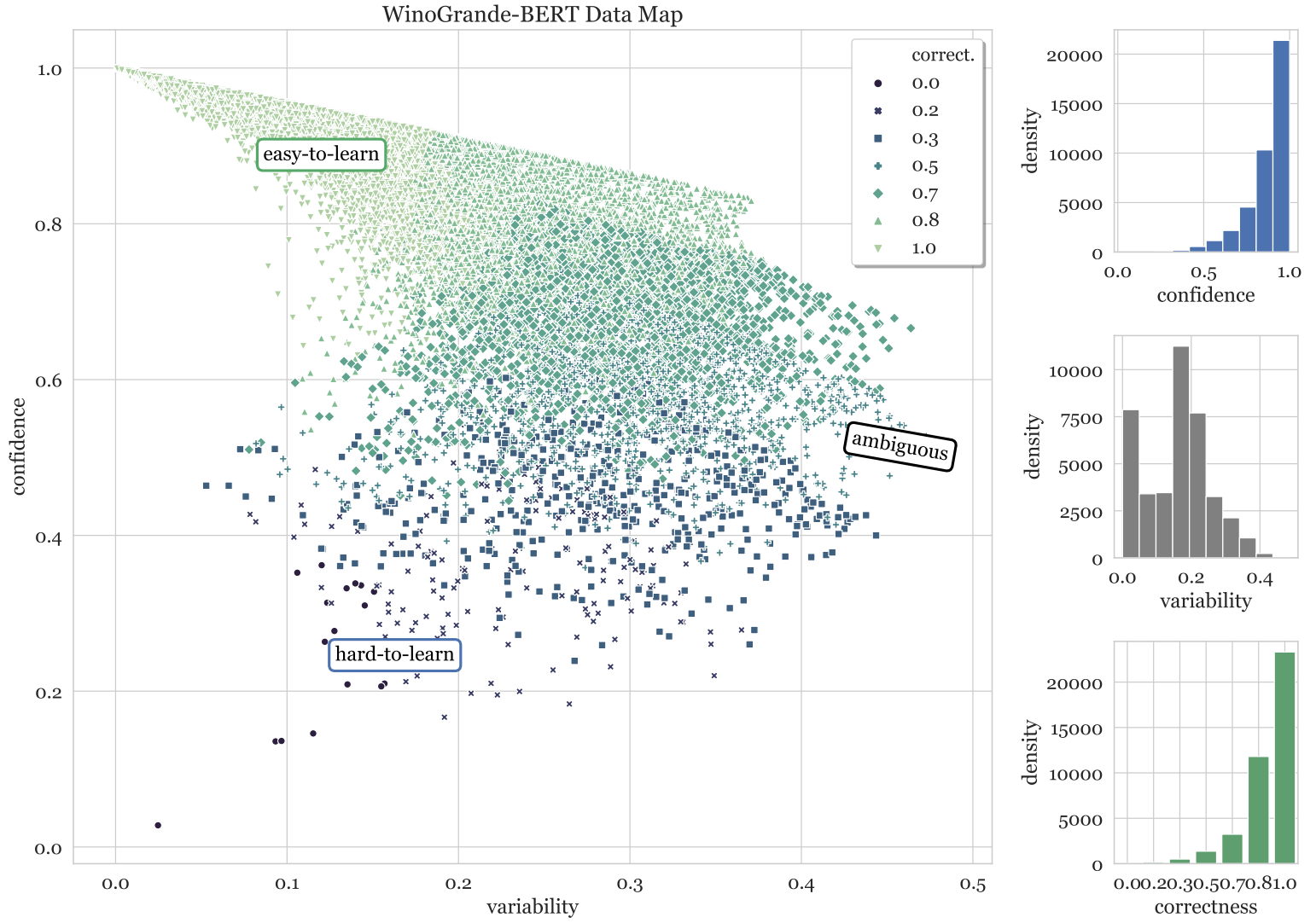

(b) Data map for WinoGrande built based on a BERT-large (Devlin et al., 2019) model. While similar regions can be seen as a WinoGrande-RoBERTA data map (Fig. 2), the densities of different regions can be different. Moreover the same instances might be mapped to different regions across maps.

Figure 9: Additional data maps, each plotting 25K instances, for clarity. 

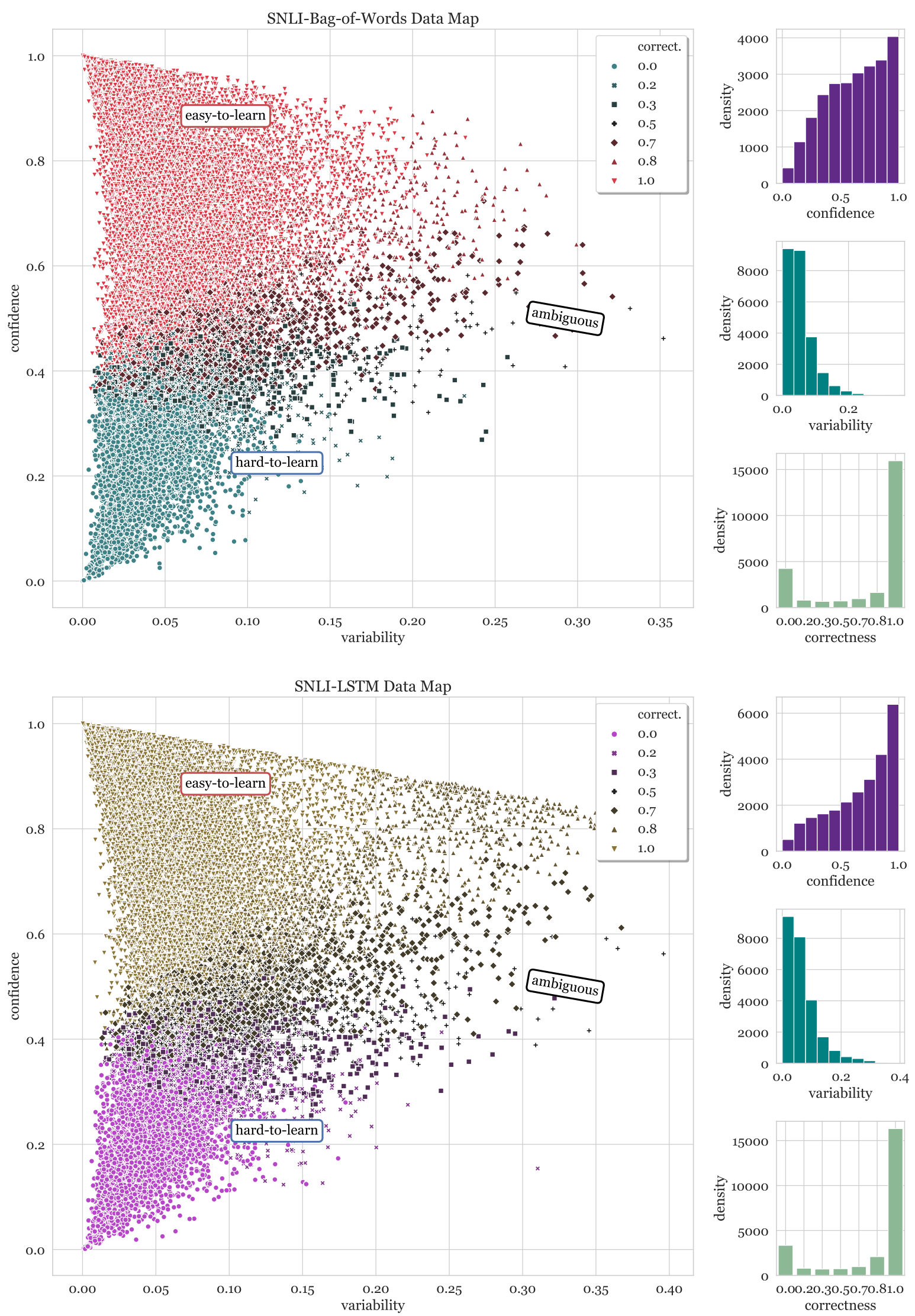

Figure 10: Data maps for SNLI based on non-RoBERTA (and weaker) architectures-bag of words (BoW; Top) and LSTMs (Bottom). Although these maps exhibit bell-shaped curves, similar to the RoBERTA data map for SNLI in $8 \mathrm{~b}$, the curvature is somewhat smaller. The spread of the data is larger across the regions, which are not as distinct as in the ROBERTA data map. These shapes could be attributed to these architectures being weaker (and hence unable to overfit to data) than those involving representations from large, pretrained language models. Each data map plots $25 \mathrm{~K}$ instances, for clarity, and are best $92.22 \mathrm{e}$ ed enlarged. 

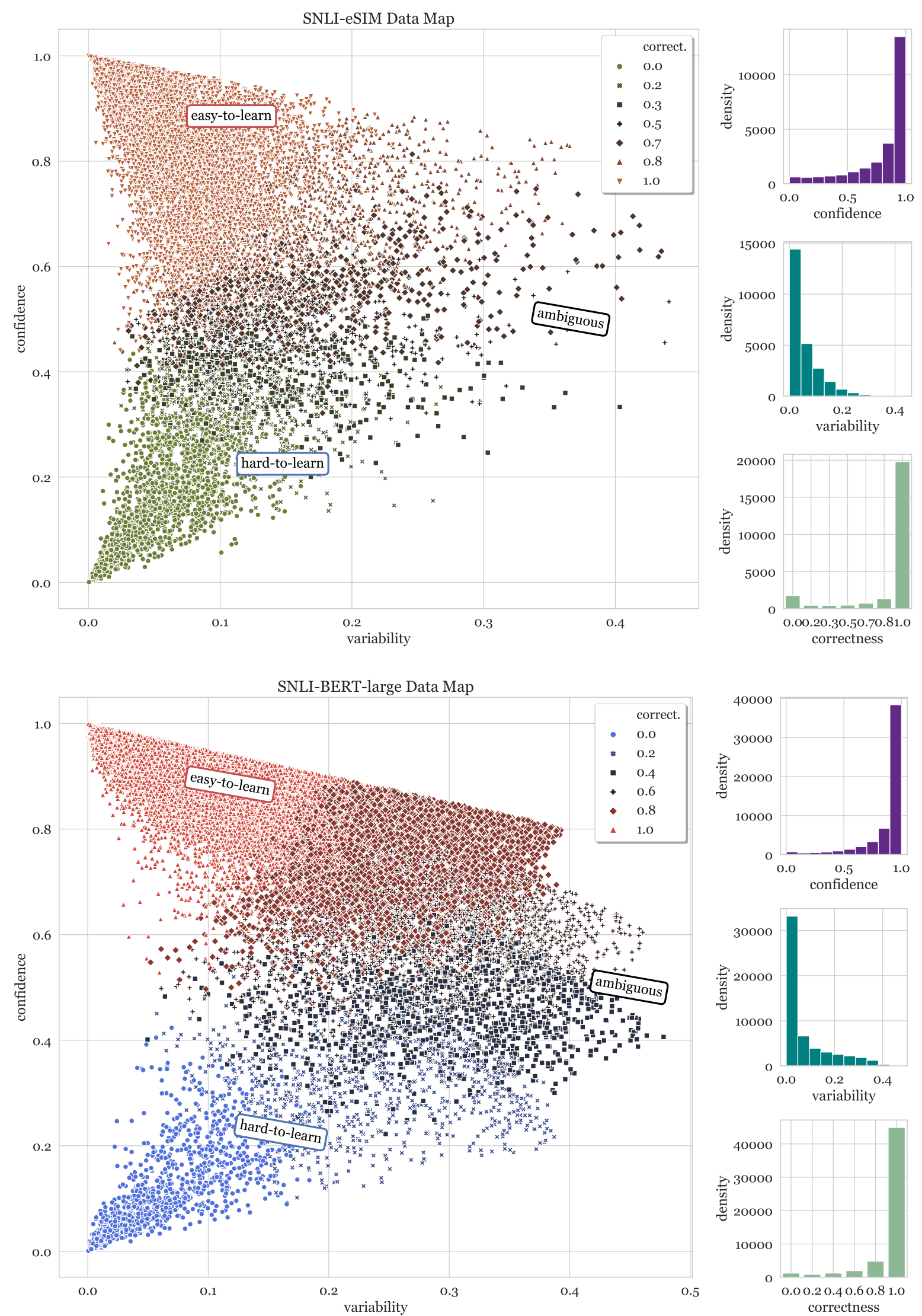

Figure 11: Data maps for SNLI based on other (weaker) architectures-bag of words eSim (Chen et al., 2017b) (Top) and BERT-large (Bottom). Although these maps exhibit bell-shaped curves, similar to the RoBERTA data map for SNLI in $8 \mathrm{~b}$, the curvature is somewhat smaller for eSIM. The spread of the data is larger across the regions, which are not as distinct as in the RoBERTA data map. Each data map plots $25 \mathrm{~K}$ instances, for clarity, and are best viewed enlarged. 